\title{
EVOLUCIÓN DE LOS PESOS Y TASA DE CRECIMIENTO DE CORDEROS PROVENIENTES DE MADRES DE BAJA CONDICIÓN CORPORAL
}

\author{
SosA, J. L. ${ }^{1}$; LeVA, P. E. ${ }^{2} ;$ Toffoli, G. D. ${ }^{2}$; Fernandez, G. B. ${ }^{1}$; \\ Boggero, C. ${ }^{1}$ \& GARCIA, M. S. ${ }^{2}$
}

\begin{abstract}
RESUMEN
El objetivo del ensayo fue evaluar el peso y el crecimiento de corderos Pampinta mantenidos al pie de la madre con una alimentación diferencial (Creep Feeding) con ovejas de condición corporal entre 1 y 1,5. El ensayo se llevó a cabo en dependencias de la Facultad de Ciencias Agrarias y Ciencias Veterinarias de la UNL, se utilizaron 20 ovejas Pampinta y 20 corderos nacidos en el mes de septiembre del 2017. La alimentación de los corderos se realizó bajo lactancia natural no restringida con alimentación diferencial. Durante la noche los corderos separados de sus madres fueron conducidos al patio de comida y recibían una ración de mezcla de soja y maíz espolvoreados con leche en polvo. Los resultados fueron analizados mediante ANOVA para un diseño completamente aleatorizado. Las variables peso al nacer $(p=0,0065)$ y tasa de crecimiento $(p=0,039)$ fueron significativamente diferentes entre sexo. La ganancia de peso diaria y el peso a los 45 días no fueron diferentes entre sexo.
\end{abstract}

Palabras clave: cordero Pampinta, alimentación diferencial.

\begin{abstract}
Evolution of weights and growth rate of lives from mothers of low body condition.

The objective of the trial was to evaluate the weight and growth of Pampinta lambs maintained at the foot of the mother with a differential feeding (Creep Feeding) with ewes of body condition 1, 1,5. The trial was carried out in units of the Faculty of Agricultural Sciences and Veterinary Sciences, 20 Pampinta sheep and 20 lambs born in the month of September of 2017. The feeding of the
\end{abstract}

1.- Facultad de Ciencias Veterinarias (UNL). Kreder 2805. (3080) Esperanza, provincia de Santa Fe. 2.- Facultad de Ciencias Agrarias (UNL). Kreder 2805. (3080) Esperanza, provincia de Santa Fe. Email: pleva@fca.unl.edu.ar Manuscrito recibido el 24 de mayo de 2018 y aceptado para su publicación el 24 de agosto de 2018.

Sosa, J. L.; Leva, P. E.; Toffoli, G. D.; Fernández, G. B.; Boggero, C. \& García, M. S. Evolución de los pesos y tasa de crecimiento de corderos provenientes de madres de baja condición corporal. FAVE - Ciencias Agrarias 17 (1): 71-74. CC BY-NC-SA 4.0 c)(1)(2) 


\section{J. L. Sosa et al.}

lambs was made under unrestricted natural lactation with differential feeding. During the night the lambs separated from their mothers were taken to the food court and received a ration of soy and corn mixture sprinkled with powdered milk.

The results were analyzed by means of ANOVA for a completely randomized design.

The variables birth weight $(p=0.0065)$ and growth rate $(p=0.039)$ were significantly different between sex, daily weight gain and weight at 45 days were not different between sex.

Key words: Lamb Pampinta, differential feeding.

\section{INTRODUCCIÓN}

La alimentación diferencial del cordero lactante conocida como Creep Feeding (CF) permite que el mismo, que se encuentra al pie de su madre, pueda acceder libremente a un concentrado (CF) de mayor valor nutritivo que el que consume su madre. El concentrado se suministra en lugares donde los corderos tienen fácil acceso, pero este queda fuera del alcance de las ovejas. Las ventajas de esta práctica: i) mejor aprovechamiento de la etapa de mayor conversión del alimento a peso vivo, particularmente músculo, ii) obtención de mayores ganancias de peso especialmente en corderos de nacimiento múltiple, iii) hacer un uso más racional y estratégico del forraje disponible y iv) favorecer la posibilidad de destete precoz $(4,6)$.

Por otro lado, la alimentación diferencial también puede ser beneficiosa cuando hay competencia entre ovejas y corderos por el forraje y/o suplementos, particularmente cuando estos recursos son escasos (Ej. períodos de escasez de forraje y de bajo valor nutritivo) o cuando existe una alta contaminación de parásitos en las pasturas (3).

El cordero a la semana de vida comienza a consumir pequeñas cantidades de alimento, tales como granos, balanceados, pasturas, etc., siendo éste el momento de mayor importancia de la suplementación, y se va incrementando a medida que la producción de leche materna disminuye, convirtiéndose posteriormente en su único alimento, independiente de la leche materna.

El objetivo del ensayo fue evaluar el peso y el crecimiento de corderos Pampinta mantenidos al pie de la madre con una alimentación diferencial (Creep Feeding).

\section{MATERIAL Y MÉTODOS}

El ensayo se realizó en dependencias de la Facultad de Ciencias Agrarias y Ciencias Veterinarias de la UNL con 20 ovejas de raza Pampinta con 20 corderos al pie, destinadas a la producción de leche. Los corderos se identificaron con caravanas plásticas en la oreja y fueron pesados dentro de las 4 hs. post-nacimiento y una vez por semana en el mismo horario previo al amamantamiento.

Al realizar el destete (45 días) se realizó el pesaje final.

Por razones de índole sanitarias (ectima, boquera) las madres disminuyeron su condición corporal, (1-1,5) por lo tanto se suspendió la temporada de ordeñe.

A los 30 días se vacunó a los corderos 
contra mancha, gangrena gaseosa y entorotoxemia, y se los desparasitó con closantel y albendazole con la finalidad de cubrir mayor espectro de vermes redondos y chatos, especialmente Haemonchus y tenías.

Los corderos permanecieron con las madres hasta el destete (45 días), y posterior en engorde a corral, hasta los $45 \mathrm{~kg}$, momento de venta como cordero pesado.

Durante el día permanecieron con las madres sobre una pastura de alfalfa de tercer año en un potrero de tres has. Por las noches, los corderos se separaban de las madres y se los alimentó con una mezcla de maíz (70\%), soja (30 \%) molida y espolvoreada con leche en polvo, para mejorar la palatabilidad. El suministro de alimento se realizó en bateas de pvc a razón de un $2 \%$ del peso promedio del lote, ajustándose quincenalmente en función del aumento de peso, observándose que al momento del suministro los primeros días quedaba un remanente pero luego comían la totalidad del mismo. Se analizaron las siguientes variables: peso al nacimiento (PN) en $\mathrm{kg}$, peso semanal (PS) en $\mathrm{kg}$, peso a los 45 días (PD) en kg, promedio de aumento de peso diario (AGD) en $\mathrm{kg}$ y tasa de crecimiento (TC) en \%.
Los resultados fueron analizados mediante ANOVA para un diseño completamente aleatorizado para las variables peso al nacimiento peso a los 45 días, ganancia de peso y tasa de crecimiento.

\section{RESULTADOS y DISCUSIÓN}

En el Cuadro1 se presentan los pesos promedios de los corderos al nacimiento, a los 45 días, la ganancia de diaria (GPD) y la tasa de crecimiento.

Se detectaron diferencias significativas en los pesos al nacimiento ( $p=0,0065)$ entre machos y hembras. Siendo los de mayores pesos los machos. Este resultado es similar a lo informado por Busetti et al., (2) para esta misma raza en sistemas pastoriles. Por otro lado, investigaciones realizadas por Leguiza et al., (5), para la misma raza, no encontraron diferencias en los pesos al nacimiento entre sexos.

En relación a la tasa de crecimiento ( $p=0,039)$, fue significativamente mayor en las hembras.

En el mismo Cuadro 1 se observa que, en relación, al peso a los 45 días y ganancia

Cuadro 1: Peso promedio al nacer, a los 45 días, la ganancia de peso diaria y tasa de crecimiento por sexo.

\begin{tabular}{|l|l|l|l|l|}
\hline & $\begin{array}{l}\text { Peso al } \\
\text { nacer }(\mathrm{kg})\end{array}$ & $\begin{array}{l}\text { Peso a los } \\
45 \text { días }(\mathrm{kg})\end{array}$ & $\begin{array}{l}\text { Ganancia de peso } \\
\text { diaria }(\mathrm{GPD}, \mathrm{kg})\end{array}$ & $\begin{array}{l}\text { Tasa de } \\
\text { crecimiento }(\mathrm{kg})\end{array}$ \\
\hline $\begin{array}{l}\text { Macho }(\mathrm{M}) \\
\mathrm{n}=11\end{array}$ & 5,61 & 16,92 & 0,251 & 2,00 \\
\hline $\begin{array}{l}\text { Hembra }(\mathrm{H}) \\
\mathrm{n}=9\end{array}$ & 4,87 & 16,59 & 0,260 & 2,4 \\
\hline EEM & 0,461 & 2,67 & 0,332 & 0,039 \\
\hline Efecto & $*$ & NS & NS & * \\
\hline
\end{tabular}

EEM error estándar de la muestra, Efecto: * = Significativo $(p<0,05)$. 


\section{J. L. Sosa et al.}

de peso diario (GPD) no se detectaron diferencias significativas entre los sexos. Estos resultados son coincidentes con los presentado por Leguiza et al., (5) para corderos Pampinta. Otros investigadores trabajando en otras razas no detectaron diferencias entre sexo en estos parámetros $(1,7)$.

\section{CONCLUSIONES}

Los corderos machos tuvieron mayores pesos al nacimiento, pero las hembras presentaron mejor tasa de crecimiento.

En ambos sexos el peso a los 45 días y las ganancias diarias de peso fueron similares.

\section{BIBLIOGRAFIA}

1.- BINACHI, G.; GARIBOTTO, G. y BENTANCUR, O. 2003. Comportamiento productivo en la progenie de carneros Ile de France y ovejas Corriedale y Polwarth. Rev. Agrociencia VII, Nº1: 9-18.

2.- BUSETTI, M. R.; SUÁREZ, V. H.; BABINEE, F. J. y BEDOTTI, D. O. 2006. Peso al nacimiento y crecimiento hasta el destete de corderos Pampinta y sus cruzas con Ile de France y Texel. RIA (INTA), 35 (2): 91-101.

3.- DOANE, T. H. 1979. Creep Feeding Lams. Univesity of Nebraska Lincoln. En: http// ianrpubs.unl.edu/sheep/ g432.htm. (Consultado 03/01/2018).

4.- GATE, N. L. 1988. Creep feeding lambs. Washington State University, College of Agriculture\&Home Economics. Extention Bulletin $\mathrm{N}^{\circ}$ 1481, 4p.

5.- LEGUIZA, H. D.; CHAGRA DIB, E. P.; EGEA, V. y SILVA COLOMER, J. 2007. Evaluación de pesos al nacimiento y ganancias de pesos hasta el destete de corderos Pampinta http://www.produccionbovina.com.ar/produccion_ovina/produccion_ovina_carne/136-Leguiza-Ovinos.pdf (consultado 31/03/2018)

6.- O'CONNELL. 2004. Early weaning of lambs. En:http//www.agri.wa.gov.au/pls/portal30/docs/folder/ikmp/lwe/adv/. (Consultado 03/01/2018).

7.- OLLETA CASTAÑER, J. L.; SAÑUDO ASTIZ, C. y SIERRA ALFRANCA, I. 1992. Producción de carne en la agrupación ovina Chrurra Tensina: calidad de la canal y de la carne en los tipos ternasco cordero de cebo. Arch. Zootec. 41: 197-208. 